\title{
Article \\ Solvability of Some Nonlocal Fractional Boundary Value Problems at Resonance in $\mathbb{R}^{n}$
}

\author{
Yizhe Feng ${ }^{+}\left(\mathbb{D}\right.$ and Zhanbing Bai ${ }^{*}+\mathbb{+}$ \\ College of Mathematics and System Science, Shandong University of Science and Technology, \\ Qingdao 266590, China; yzfeng2021@163.com \\ * Correspondence: zhanbingbai@163.com \\ † These authors contributed equally to this work.
}

\begin{abstract}
In this paper, the solvability of a system of nonlinear Caputo fractional differential equations at resonance is considered. The interesting point is that the state variable $x \in \mathbb{R}^{n}$ and the effect of the coefficient matrices matrices $B$ and $C$ of boundary value conditions on the solvability of the problem are systematically discussed. By using Mawhin coincidence degree theory, some sufficient conditions for the solvability of the problem are obtained.
\end{abstract}

Keywords: coincidence degree theory; four-point boundary value problem system; at resonance

Citation: Feng, Y.; Bai, Z. Solvability of Some Nonlocal Fractional Boundary Value Problems at Resonance in $\mathbb{R}^{n}$. Fractal Fract. 2022, 6, 25. https://doi.org/10.3390/ fractalfract6010025

Academic Editor: Rodica Luca

Received: 12 December 2021 Accepted: 31 December 2021 Published: 1 January 2022

Publisher's Note: MDPI stays neutral with regard to jurisdictional claims in published maps and institutional affiliations.

Copyright: (C) 2022 by the authors. Licensee MDPI, Basel, Switzerland. This article is an open access article distributed under the terms and conditions of the Creative Commons Attribution (CC BY) license (https:// creativecommons.org/licenses/by/ $4.0 /)$.

\section{Introduction}

In partial differential equations theory, multipoint boundary conditions are those which the solutions of multiple-parameter differential equations should satisfy. In recent decades, more and more mathematicians turned their attention to nonlinear boundary value problems (BVPs) in resonance cases and non-resonance cases. For some non-resonance cases, we recommend readers to [1-4], and for resonance cases to [5-12] and the references therein. In [8], Feng first obtained the existence of one solution of semilinear three-point BVPs at resonance by making use of the coincidence degree theory of Mawhin. Then, as an extension of [8], Ma [9] first developed the upper and lower solution method to obtain some multiplicity results. Motivated by [9], Bai [6] researched a four-point boundary value problem, and proved the existence and multiplicity results by making use of the method of upper and lower solutions established by the coincidence degree theorem. Subsequently, various boundary value conditions were studied.

V.A. Il'in and E.I. Moiseev in [1] studied Sturm-Liouville operator of the first kind of nonlocal boundary value problem, which originated from the famous work of A. V. Bitsadze and A. A. Samarskogo [3]: In the Euclidean $n$-dimensional space with orthogonal Cartesian coordinates $x_{1}, x_{2}, \ldots, x_{n}$, the elliptic linear differential equation on the $(n-1)$ -dimensional piecewise smooth Lyapunov surface is transformed into a nonlocal problem of an ordinary differential equation when solving a partial differential equation by the separation of variables method. When the state variable is $n$-dimensional, consideration of the general fractional model will naturally involve the model of the problem considered in this paper.

To our best knowledge, before P.D. Phung [13], almost all articles on resonance BVPs were focused on a single second-order equation with the dimension of Ker $L \in[0,2]$. For a second-order equation boundary value problem system with $x \in \mathbb{R}^{n}$, the dimension of Ker $L$ will be between 0 and $2 n$; it will not be as easy as $\operatorname{dim} \operatorname{Ker} L=1$ to establish projections $Q$ for matrices $B$ and $C$ with different properties. For the case of $n=2$, Zhang in [12] considered a three-point BVP at resonance for nonlinear fractional differential equations:

$$
\left\{\begin{array}{l}
D_{0+}^{\alpha} u(t)=f\left(t, v(t), D_{0+}^{\beta-1} v(t)\right), \quad 0<t<1, \\
D_{0+}^{\beta} v(t)=g\left(t, u(t), D_{0+}^{\alpha-1} u(t)\right), \quad 0<t<1, \\
u(0)=v(0)=0, \quad u(1)=\sigma_{1} u\left(\eta_{1}\right), \quad v(1)=\sigma_{2} v\left(\eta_{2}\right),
\end{array}\right.
$$


and obtained two existence results using the coincidence degree theory. In [13]. P.D. Phung first researched the following resonant three-point BVPs in $\mathbb{R}^{n}$ :

$$
\left\{\begin{array}{l}
x^{\prime \prime}(t)=f\left(t, x, x^{\prime}\right), \quad t \in(0,1), \\
x^{\prime}(0)=\theta, \quad x(1)=A x(\eta),
\end{array}\right.
$$

where $\theta$ is an $n$-order zero vector, the matrix $A$ satisfies one of the following conditions:

$$
\left\{\begin{array}{l}
A^{2}=I(\text { stands for } n-\text { order identity matrix }), \\
A^{2}=A
\end{array}\right.
$$

In [14], P.D. Phung removed the restriction on matrix $A$ and studied the solvability of the same problem as in [13]. Then, P.D. Phung [15] used similar methods to study the following three-point boundary conditions in the fractional differential equations at resonance:

$$
\begin{gathered}
D^{\alpha} x(t)=f\left(t, x(t), D^{\alpha-1} x(t),\right. \\
x(0)=\theta, D^{\alpha-1} x(1)=A D^{\alpha-1} x(\eta) .
\end{gathered}
$$

Recently, the solvability of integer or fractional differential equations with a wide range of boundary value conditions at resonance in $\mathbb{R}^{n}$ has been researched. We direct readers to [13-21] for details.

For nearly a decade, the resonant boundary value problem with $n$ equations has been studied by an increasing number of mathematicians. However, we found that the following two problems have not been addressed. First, Zhang in [12] studied the resonance boundary value problem of two equations, but used the same boundary value conditions for different state variables $u$ and $v$, so the study was similar to that of a single equation and could not be easily extended to the case of $n$ dimensions. Therefore, in this study we consider the characterization of different constraints on different state variables, in other words, we introduce matrices to control the constraints on state variables so that the expression of the equation can be richer. However, other works $[13-16,20,21]$ under the condition of zero boundary value (similar to $u(0)=0$ ) studied $n$ equations of the problem. Gupta in [10] proposed that many multi-point boundary value problems can be transformed into four-point boundary value problems under certain conditions, so studying four-point BVPs is more meaningful. The four-point boundary value condition does not contain zero boundary value, which makes the structure of irreversible operators and the construction of projection $P$ and $Q$ more complicated than that of three-point BVPs. Therefore, it is more meaningful to introduce a matrix to study four-point boundary value problems in mathematics.

Motivated by the above ideas, we consider the following fractional-order equations with a new boundary value condition in $\mathbb{R}^{n}$ :

$$
\begin{gathered}
{ }^{c} D_{0+}^{\alpha} u(t)=f\left(t, u(t),{ }^{c} D_{0+}^{\alpha-1} u\right), \quad t \in(0,1), \\
u(0)=B u(\xi), \quad u(1)=C u(\eta),
\end{gathered}
$$

where $0<\eta, \xi<1,1<\alpha \leqslant 2 ; B, C$ are two $n$-order nonzero square matrices, ${ }^{c} D_{0+}^{\alpha}$ represents the Caputo differentiation, and $f:[0,1] \times \mathbb{R}^{2 n} \rightarrow \mathbb{R}^{n}$ satisfies Carathéodory conditions. In this situation, Ker $L$ may become a polynomial set with vector coefficients and the construction of projectors will be somewhat complex. We say $f:[0,1] \times \mathbb{R}^{2 n} \rightarrow \mathbb{R}^{n}$ satisfies Carathéodory conditions, that is,

(A1) $f(\cdot, u, v)$ is measurable on $[0,1]$ for all $(u, v) \in \mathbb{R}^{n} \times \mathbb{R}^{n}$.

(A2) $f(s, \cdot, \cdot)$ is continuous on $\mathbb{R}^{n} \times \mathbb{R}^{n}$, for a.e. $s \in[0,1]$.

(A3) The function $g_{W}(t)=\sup _{(u, v) \in \bar{W}}|f(s, u, v)|$ is Lebesgue integrable on $0 \leqslant s \leqslant 1$ for all compact set $W \subset \mathbb{R}^{n} \times \mathbb{R}^{n}$. 
The problem in (1) and (2) is in resonance, meaning that the following linear homogeneous boundary value problem has nontrivial solutions:

$$
\begin{gathered}
{ }^{C} D_{0+}^{\alpha} u(t)=\theta, \quad 0<t<1, \\
u(0)=B u(\xi), \quad u(1)=C u(\eta) .
\end{gathered}
$$

By (3), there is $u(t)=c_{1}+c_{2} t, c_{1}, c_{2} \in \mathbb{R}^{n}$. Combining with (4), we can get the following equations:

$$
\left\{\begin{array}{l}
(I-\eta C) c_{1}+(I-C) c_{2}=\theta, \\
-\xi B c_{1}+(I-B) c_{2}=\theta .
\end{array}\right.
$$

Clearly, the resonance condition is

$$
\Delta=\left|\begin{array}{cc}
I-\eta C & I-C \\
-\xi B & I-B
\end{array}\right|=0
$$

From the calculation formula of block matrix determinant, we can know that $\Delta=0$ if and only if

$$
|(I-\eta C)(I-B)+\xi B(I-C)|=0 .
$$

Condition (5) can be divided into three cases:

Case $(1) B \neq I, C \neq I,|(I-\eta C)(I-B)+\xi B(I-C)|=0$;

Case (2) $B=I,|I-C|=0$;

Case (3) $B \neq I, C=I,|I-B|=0$.

The paper is organized as follows. In Section 2, we state several notations and definitions. In Sections 3 and 4, two main theorems (see Theorem 2 and 3) are established for the solvability of problem (1) and (2) under resonance cases (1) and (2), respectively. It is worth mentioning that, inspired by [14], in Section 4, we remove the restriction on the matrix $C$, and give the existence theorem of the solution of the problem only under the most basic resonance conditions (refer to case (2)).

\section{Preliminaries}

First, we recall some related definitions and lemmas of fractional calculus; we refer the readers to [22] for more properties.

Definition 1. The $\alpha$-order $(\alpha>0)$ Riemann-Liouville fractional integral of function $u$ is defined as

$$
I_{0+}^{\alpha} u(t)=\frac{1}{\Gamma(\alpha)} \int_{0}^{t} \frac{u(s)}{(t-s)^{1-\alpha}} d s,
$$

and the right side of the equation is defined at $(0, \infty)$.

Definition 2. The $\alpha$-order $(\alpha>0)$ Caputo fractional derivative of function $u: R_{+} \rightarrow R$ is defined as

$$
{ }^{c} D_{0+}^{\alpha} u(t)=I_{0+}^{n-\alpha} D^{n} u(t)=\frac{1}{\Gamma(n-a)} \int_{0}^{t} \frac{u^{(n)}(s)}{(t-s)^{1+\alpha-n}} d s
$$

as long as the right side of the equation is defined at $(0, \infty)$.

Lemma 1 ([22]). If $u \in C^{n-1}(0,1) \cap L[0,1]$, then the fractional differential equation

$$
{ }^{C} D_{0+}^{\alpha} u(t)=0
$$

has a unique solution

$$
u(t)=\sum_{i=0}^{n-1} \frac{u^{(i)}(0)}{i !} t^{k}
$$


The following lemma is also very important for subsequent research.

Lemma 2 ([22]). Let $\alpha>0$ and $n-1<\alpha \leqslant n$.

(1) Let $\alpha>\theta>0$ and $u$ be a continuous function, then

$$
{ }^{C} D_{0+}^{\theta} I_{0+}^{\alpha} u(t)=I_{0+}^{\alpha-\theta} u .
$$

(2) Let $u$ be an absolute continuous function of $n-1$ times differentiable, then

$$
I_{0+}^{\alpha}{ }^{C} D_{0+}^{\alpha} u(t)=u(t)-\sum_{i=0}^{n-1} \frac{D^{i} u(0)}{i !} t^{i} .
$$

Let $X, Y$ be two Banach spaces, we call $L: \operatorname{dom} L \subset X \rightarrow Y$ a Fredholm mapping of index zero if

(E1) Im $L$ is closed in $Y$ and has codimension of finite dimension;

(E2) Tthe dimension of Ker $L$ is equal to the codimension of $\operatorname{Im} L$.

If $L$ satisfies (E1) and (E2), then there will be two projectors $Q: Y \rightarrow Y, P: X \rightarrow X$ satisfies $\operatorname{Ker} Q=\operatorname{Im} L, \operatorname{Im} P=\operatorname{Ker} L$. Therefore, we can get the straight-sum decomposition: $Y=\operatorname{Im} L \oplus \operatorname{Im} Q, X=\operatorname{Ker} L \oplus \operatorname{Ker} P$. Here, by $K_{P}$ we denote the inverse of $\left.L\right|_{\text {Ker } P \cap \text { dom } L}$ : Ker $P \cap \operatorname{dom} L \rightarrow \operatorname{Im} L$ and by $K_{P, Q}:=K_{P}(I d-Q)$ the generalized inverse of $L$.

We call $N L$-compact on $\bar{\Omega}(\Omega$ is an open bounded subset of $X$ with $\operatorname{dom} L \cap \Omega \neq \varnothing$, when it satisfies

(F1) $Q N(\bar{\Omega})$ is bounded;

(F2) $K_{P}(I d-Q) N: \bar{\Omega} \rightarrow X$ is completely continuous.

Theorem 1 ([23]). Let $L$ be a Fredholm operator of index zero and $N(\bar{\Omega})$ be L-compact. Suppose the following conditions are satisfied:

(i) $L u \neq \lambda N u$ for all $x \in \partial \Omega \cap(\operatorname{dom} L \backslash$ Ker $L)$ and $0<\lambda<1$;

(ii) $N u \notin \operatorname{Im} L$ for all $x \in \partial \Omega \cap \operatorname{Ker} L$;

(iii) $\operatorname{deg}\left(\left.\mathcal{J} Q N\right|_{\Omega} \cap \operatorname{Ker} L^{\prime}, \operatorname{Ker} L \cap \Omega, 0\right) \neq 0$, where $\mathcal{J}: \operatorname{Im} Q \rightarrow \operatorname{Ker} L$ is an isomorphism, and $Q: Y \rightarrow Y$ is a projection as above.

Then, the equation $L u=N u$ has at least one solution in $\operatorname{dom} L \cap \bar{\Omega}$.

By $\|u\|=\max \left\{\|u\|_{\infty},\left\|{ }^{c} D_{0+}^{\alpha-1} u\right\|_{\infty}\right\}$ we denote the norm of space $X=C^{1}\left([0,1] ; \mathbb{R}^{n}\right)$, where $\|\cdot\|_{\infty}$ is the maximum norm. Additionally, by $\|y\|_{1}$ we denote the Lebesgue norm of $Y=L^{1}\left([0,1] ; \mathbb{R}^{n}\right)$. Set

$$
X_{1}:=\left\{u:[0,1] \rightarrow \mathbb{R}^{n} \mid u \in C^{2}\left([0,1] ; \mathbb{R}^{n}\right)\right\}
$$

Then, define map $L: \operatorname{dom} L \rightarrow Y$ by setting

$$
\operatorname{dom} L=\left\{u \in X_{1}: u(0)=B u(\xi), u(1)=C u(\eta)\right\},
$$

for $u \in \operatorname{dom} L$,

$$
L u:={ }^{C} D_{0+}^{\alpha} u
$$

\section{Existence Results for Case (1)}

Now, we show the solvability of BVP (1), (2) when $B \neq I, C \neq I, \mid(I-\eta C)(I-B)+$ $\xi B(I-C) \mid=0$. Furthermore, suppose the matrices $B, C$ satisfy the following conditions:

(H1) $I-B$ is reversible;

(H2) $(\eta C-I) \xi^{\alpha-1}-\eta^{\alpha} C+I$ is reversible;

(H3) $I-\eta C+\xi(I-C)(I-B)^{-1} B=\Theta$, 
where $\Theta$ is an $n$-order zero matrix. From (12) we can know

$$
\operatorname{Ker} L=\left\{c_{2} t+C_{0} c_{2}, c_{2} \in \mathbb{R}^{n}\right\}
$$

where $C_{0}=\xi(I-B)^{-1} B$, and from $(\mathrm{H} 3)$ we have $(I-C) C_{0}=(\eta C-I)$. Let

$$
G(s)= \begin{cases}(\xi-s)^{\alpha-1}(I-C)(I-B)^{-1} B-(\eta-s)^{\alpha-1} C+(1-s)^{\alpha-1} I, & 0 \leqslant s \leqslant \xi ; \\ -(\eta-s)^{\alpha-1} C+(1-s)^{\alpha-1} I, & \xi<s<\eta ; \\ (1-s)^{\alpha-1} I, & \eta \leqslant s \leqslant 1,\end{cases}
$$

then

$$
\operatorname{Im} L=\left\{y \in Y \mid \frac{1}{\Gamma(\alpha)} \int_{0}^{1} G(s) y(s) d s=\theta\right\} .
$$

Define a mapping $Q: Y \rightarrow Y$ as

$$
Q y=\gamma \int_{0}^{1} G(s) y(s) d s
$$

where

$$
\gamma=\alpha\left\{(\eta C-I) \xi^{\alpha-1}-\eta^{\alpha} C+I\right\}^{-1}
$$

Lemma 3. The operator $L$ is a Fredholm operator with an index of zero.

Proof. For $y \in Y, \forall t \in[0,1]$

$$
\begin{aligned}
Q^{2} y(t) & =\gamma \int_{0}^{1} G(s) Q y(s) d s \\
& =\frac{\gamma}{\alpha}\left\{(\eta C-I) \xi^{\alpha-1}-\eta^{\alpha} C+I\right\} Q y(t) \\
& =Q y(t)
\end{aligned}
$$

so linear operator $Q$ is a continuous projector. For $y \in \operatorname{Im} L$, one has $Q y(t)=\theta$; this shows that $y \in \operatorname{Ker} Q$. In fact, $\operatorname{Im} L=\operatorname{Ker} Q$.

Let $y \in Y$ and it is easy to verify $y-Q y \in \operatorname{Im} L$. Thus, $Y=\operatorname{Im} L+\operatorname{Im} Q$. For every $y \in \operatorname{Im} Q$ have the form $y=c, c \in \mathbb{R}^{n}$. At this time, if $y \in \operatorname{Im} L$, then $y=\theta$. Hence, $Y=\operatorname{Im}$ $L \oplus \operatorname{Im} Q$. Combine with codim $\operatorname{Im} L=\operatorname{dim} \operatorname{Im} Q=\operatorname{dim} \operatorname{Ker} L$, so $L$ satisfies (E1) and (E2), and the index of the Fredholm operator $L$ is zero.

Define another projector $P: X \rightarrow X$ by

$$
P u=u^{\prime}(0) t+C_{0} u^{\prime}(0)
$$

For $v \in \operatorname{Ker} L$, one has

$$
v(t)=c_{2} t+C_{0} c_{2}, c_{2} \in \mathbb{R}^{n},
$$

and

$$
P v(t)=c_{2} t+C_{0} c_{2}=v(t) .
$$

This shows that $v \in \operatorname{Im} P$. Conversely, for every $v \in \operatorname{Im} P$, there is $x \in X$ such that $v(t)=P x(t)$. Thus,

$$
v(t)=P x(t)=x^{\prime}(0) t+c_{0} x^{\prime}(0) \in \operatorname{Ker} L .
$$

Hence, Ker $L=\operatorname{Im} P$. Clearly, $X=\operatorname{Ker} P \oplus \operatorname{Ker} L$. In fact, Ker $P \cap \operatorname{Ker} L=\{\theta\}$.

Define a mapping $K_{P}: \operatorname{Im} L \rightarrow \operatorname{Ker} P \cap \operatorname{dom} L$ as

$$
K_{P} y(t)=(I-B)^{-1} B I_{0+}^{\alpha} y(\xi)+I_{0+}^{\alpha} y(t), 0 \leqslant t \leqslant 1 .
$$


Lemma 4. $K_{P}$ is the inverse of the mapping $\left.L\right|_{\text {Ker } P \cap \operatorname{dom} L}$ and

$$
\left\|K_{P} y\right\| \leqslant D\|y\|_{1}
$$

where $D=1+\xi\left\|(I-B)^{-1} B\right\|_{*},\|\cdot\|_{*}$ stand for the max-norm of matrices.

Proof. Let $y \in \operatorname{Im} L$. It is clear that $K_{P} y(0)=B K_{P} y(\xi)$ and $K_{P} y(1)=C K_{P} y(\eta)$, such that $K_{P} y \in \operatorname{dom} L$. Furthermore

$$
P K_{P} y(t)=\left.\left(K_{P} y\right)^{\prime}(t)\right|_{t=0} t+\left.c_{0}\left(K_{P} y\right)^{\prime}(t)\right|_{t=0}=\theta .
$$

This shows that $K_{P} y \in \operatorname{Ker} P$. So, the definition of $K_{P}$ is reasonable.

For $u \in \operatorname{Ker} P \cap \operatorname{dom} L$, from (11), one has

$$
\begin{aligned}
K_{P} L u & =(I-B)^{-1} B I_{0+}^{\alpha}{ }^{c} D_{0+}^{\alpha} u(\xi)+I_{0+}^{\alpha}{ }^{c} D_{0+}^{\alpha} u(t) \\
& =(I-B)^{-1} B\left[u(\xi)-u(0)-u^{\prime}(0) \xi\right]-I\left(u(0)-u(t)+u^{\prime}(0) t\right) \\
& =u .
\end{aligned}
$$

Conversely, for $y \in \operatorname{Im} L$, one has $L K_{P} y=y$. Thus, $K_{P}=\left(\left.L\right|_{\operatorname{dom} L \cap \operatorname{Ker} P}\right)^{-1}$.

Again, since

$$
\begin{aligned}
\left\|{ }^{c} D_{0+}^{\alpha-1}\left(K_{P} y\right)(t)\right\|_{\infty} & =\left\|(I-B)^{-1} B I_{0+}^{1} y(\xi)\right\|_{\infty}+\left\|I_{0+}^{1} y(t)\right\|_{\infty} \\
& \leqslant\left(1+\xi\left\|(I-B)^{-1} B\right\|_{*}\right)\|y(t)\|_{1},
\end{aligned}
$$

combining with (16), one has

$$
\left\|K_{P} y\right\|_{\infty} \leqslant \frac{D}{\Gamma(\alpha)}\|y\|_{1} .
$$

Thus, we have $\left\|K_{P} y\right\| \leqslant D\|y\|_{1}$.

Define an operator $N: X \rightarrow Y$ by

$$
N u(t)=f\left(t, u(t),{ }^{c} D_{0+}^{\alpha-1} u(t)\right), 0 \leqslant t \leqslant 1 .
$$

Lemma 5. $N$ is L-compact.

Proof. We divide the proof into two parts. The first part is bounded continuous. The second part is completely continuous. Indeed, for $f\left(t, u(t),{ }^{c} D_{0+}^{\alpha-1} u(t)\right)$, there exists a function $g_{W}(t): R \rightarrow Y$ s.t. for every $u \in W \subset X$ and a.e. $0 \leqslant t \leqslant 1$

$$
\left\|f\left(t, u,{ }^{c} D_{0+}^{\alpha-1} u\right)\right\|_{\infty} \leqslant g_{W} .
$$

Combining with (13), one has

$$
\|Q y\|_{1} \leqslant\|G(s)\|_{*}\|\gamma\|_{*}\|y\|_{1}
$$

where

$$
\begin{aligned}
\|\gamma\|_{*} & =\alpha\left\|\left\{(\eta C-I) \xi^{\alpha-1}-\eta^{\alpha} C+I\right\}^{-1}\right\|_{*,} \\
\|G(s)\|_{*} & =\left(1+\|C\|_{*}+\left\|(I-C)(I-B)^{-1} B\right\|_{*}\right) .
\end{aligned}
$$

Thus, $Q N(\bar{W})$ is bounded. Obviously, $Q N(W)$ is continuous.

For all $u \in W \subset X$, one has

$$
\begin{aligned}
K_{P, Q} N u & =K_{P}(I-Q) N u \\
& =(I-B)^{-1} B I_{0+}^{\alpha} N u(\xi)+I_{0+}^{\alpha} N u(t)-(I-B)^{-1} B I_{0+}^{\alpha} Q N u(\xi)-I_{0+}^{\alpha} Q N u(t) \\
& =(I-B)^{-1} B I_{0+}^{\alpha} N u(\xi)+I_{0+}^{\alpha} N u(t)
\end{aligned}
$$




$$
\begin{gathered}
-\frac{\gamma}{\Gamma(\alpha)}\left\{\xi^{\alpha}(I d-B)^{-1} B \int_{0}^{1} G(s) N u(s) d s+t^{\alpha} \int_{0}^{1} G(s) N u(s) d s\right\} . \\
{ }^{c} D_{0+}^{\alpha-1} K_{P, Q} N u={ }^{c} D_{0+}^{\alpha-1} K_{P}(I d-Q) N u=I_{0+}^{1} N x(t)-\gamma \Gamma(\alpha+1) t \int_{0}^{1} G(s) N u(s) d s .
\end{gathered}
$$

Combining (20), (22), and (23), we have

$$
\begin{aligned}
&\left|K_{P, Q} N u(t)\right| \leqslant\left(1+\left\|(I-B)^{-1} B\right\|_{*}\right)\|N u\|_{1} \\
&+\frac{\|\gamma\|_{*}}{\Gamma(\alpha)}\left(1+\left\|(I-B)^{-1} B\right\|_{*}\right)\|G(s)\|_{*}\|N u\|_{1}, \\
&\left|{ }^{c} D_{0+}^{\alpha-1} K_{P, Q} N u(t)\right| \leqslant\left(1+\Gamma(\alpha+1)\|\gamma\|_{*}\|G(s)\|_{*}\right)\|N u\|_{1} .
\end{aligned}
$$

That is, $K_{P, Q} N u(W)$ is uniformly bounded in $X$. Now we only need to prove $K_{P, Q} N u(W)$ is equicontinuous in $X$ to end the proof of Lemma 5 . For $0 \leqslant t_{1}<t_{2} \leqslant 1$, one has

$$
\begin{aligned}
& \left|K_{P, Q} N u\left(t_{2}\right)-K_{P, Q} N u\left(t_{1}\right)\right| \\
& \leqslant \frac{1}{\Gamma(\alpha)}\left|\int_{t_{1}}^{t_{2}}\left(t_{2}-s\right)^{\alpha-1} N u(s) d s+\int_{0}^{t_{1}}\left(\left(t_{2}-s\right)^{\alpha-1}-\left(t_{1}-s\right)^{\alpha-1}\right) N u(s) d s\right| \\
& \quad+\frac{\gamma}{\alpha \Gamma(\alpha)}\|G(s)\|_{*}\|N u\|_{1}\left|t_{2}^{\alpha}-t_{1}^{\alpha}\right| \\
& \leqslant \\
& \quad \frac{1}{\Gamma(\alpha)}\left(\int_{0}^{t_{1}}\left(t_{2}-t_{1}\right)^{\alpha-1} g_{W}(s) d s+\int_{t_{1}}^{t_{2}} g_{W}(s) d s\right) \\
& \quad+\frac{\gamma}{\alpha \Gamma(\alpha)}\|G(s)\|_{*}\left\|g_{W}(t)\right\|_{1}\left|t_{2}^{\alpha}-t_{1}^{\alpha}\right|
\end{aligned}
$$

and

$$
\left|{ }^{c} D_{0+}^{\alpha-1} K_{P, Q} N u\left(t_{2}\right)-{ }^{c} D_{0+}^{\alpha-1} K_{P, Q} N u\left(t_{1}\right)\right| \leqslant \int_{t_{1}}^{t_{2}} g_{W}(s) d s+\gamma\|G(s)\|_{*}\left\|g_{W}(t)\right\|_{1}\left|t_{2}-t_{1}\right| .
$$

Thus, $K_{P, Q} N u(W)$ is equicontinuous in $\mathrm{X}$. In summary, $N$ is $L$-compact.

We will use the following assumptions:

(M1) For all $t \in[0,1], x, y \in \mathbb{R}^{n}$, there exist three functions $a_{1}, b_{1}, c \in Y$, s.t.

$$
\left(1+\left\|c_{0}\right\|_{*}+D\right)\left(\left\|a_{1}\right\|_{1}+\left\|b_{1}\right\|_{1}\right)<1,
$$

and

$$
|f(t, x, y)| \leqslant a_{1}(t)|x|+b_{1}(t)|y|+c(t)
$$

where $D$ is the constant given in (17).

(M2) For $u \in \operatorname{dom} L$, if there exist $\sigma_{1} \in R_{+}$, s.t.

$$
\left|{ }^{c} D_{0+}^{\alpha-1} u(v)\right|>\sigma_{1}, \forall v \in[0,1],
$$

then

$$
\begin{array}{rl}
C \int_{0}^{\eta}(\eta-s)^{\alpha-1} & f\left(v, u(v),{ }^{c} D_{0+}^{\alpha-1} u(v)\right) d v \\
& -I \int_{0}^{1}(1-s)^{\alpha-1} f\left(v, u(v) d v,{ }^{c} D_{0+}^{\alpha-1} u(v)\right) d v \in \operatorname{Im}(I-C) .
\end{array}
$$

(M3) Let $q(t):=\left(t I+C_{0}\right), C_{0}=\xi(I-B)^{-1} B$, and

$$
q(t) \tau=\left(q_{1}, \ldots, q_{n}\right)^{\top}, q_{i} \in \mathbb{R} .
$$


If there exist $\sigma_{2} \in R_{+}$, s.t. $\forall t \in[0,1]$,

$$
\left|q_{i}\right|>\sigma_{2}, \forall \tau \in \mathbb{R}^{n}, i=1, \ldots, n,
$$

then either

$$
\langle q(t) \tau, Q N(q(t) \tau)\rangle \leqslant 0 \text { or }\langle q(t) \tau, Q N(q(t) \tau)\rangle \geqslant 0,
$$

$\langle\cdot, \cdot\rangle$ stands for the scalar product in $\mathbb{R}^{n}$.

Theorem 2. If assumptions (M1)-(M3) are satisfied, then Problem (1), (2) has at least one solution in $X$.

Proof. Set $\Omega_{1}=\{x \in \operatorname{dom} L \backslash \operatorname{Ker} L: L x=\lambda N x, 0<\lambda<1\}$. For $u \in \Omega_{1}$, one has $N u \in \operatorname{Im} L=\operatorname{Ker} Q$. Thus,

$$
\begin{aligned}
C \int_{0}^{\eta}(\eta-s)^{\alpha-1} f\left(s, u(s),{ }^{c} D_{0+}^{\alpha-1} u(s)\right) d s-I \int_{0}^{1}(1-s)^{\alpha-1} f\left(s, u(s),{ }^{c} D_{0+}^{\alpha-1} u(s)\right) d s \\
=(I-C)(I-B)^{-1} B \int_{0}^{\xi}(\xi-s)^{\alpha-1} f\left(s, u(s),{ }^{c} D_{0+}^{\alpha-1} u(s)\right) d s \in \operatorname{Im}(I-C) .
\end{aligned}
$$

From (M2), there exist $t_{0} \in[0,1]$, s.t. $\left|{ }^{c} D_{0+}^{\alpha-1} u\left(t_{0}\right)\right| \leqslant \sigma_{1}$, thus

$$
\left|{ }^{c} D_{0+}^{\alpha-1} u(0)\right|=\left|{ }^{c} D_{0+}^{\alpha-1} u\left(t_{0}\right)-\int_{0}^{t_{0}}{ }^{c} D_{0+}^{\alpha} u(s) d s\right| \leqslant \sigma_{1}+\left\|{ }^{c} D_{0+}^{\alpha} u(t)\right\|_{1} .
$$

Furthermore

$$
\|P u(t)\|=\left\|u^{\prime}(0) t+C_{0} u^{\prime}(0)\right\| \leqslant\left(\|N u\|_{1}+\sigma_{1}\right)\left(1+\left\|C_{0}\right\|_{*}\right) .
$$

Note that $I d$ is the identity operator. Combining with (27), one has

$$
\begin{aligned}
\|u(t)\| & =\|P u+(I d-P) u\| \\
& \leqslant\|P u\|+\left\|K_{P} L(I d-P) u\right\| \\
& \leqslant\left(\|N u\|_{1}+\sigma_{1}\right)\left(1+\left\|C_{0}\right\|_{*}\right)+D\|N u\|_{1} \\
& =\left(1+\left\|C_{0}\right\|_{*}+D\right)\|N u\|_{1}+\left(1+\left\|C_{0}\right\|_{*}\right) \sigma_{1},
\end{aligned}
$$

where $D$ was given in (16). Combining (19), (28), and (M1), we get

$$
\begin{aligned}
\|N u\|_{1} & \leqslant \int_{0}^{1}\left|f\left(s, u(s),{ }^{c} D_{0+}^{\alpha-1} u(s)\right)\right| d s \\
& \leqslant\left\|a_{1}\right\|_{1}\|u\|_{\infty}+\left\|b_{1}\right\|_{1}\left\|^{c} D_{0+}^{\alpha-1} u\right\|_{\infty}+\|c\|_{1} \\
& \leqslant\left(\left\|a_{1}\right\|_{1}+\left\|b_{1}\right\|_{1}\right)\|u\|+\|c\|_{1} \\
& \leqslant\left(\left\|a_{1}\right\|_{1}+\left\|b_{1}\right\|_{1}\right)\left[\left(1+\left\|C_{0}\right\|_{*}+D\right)\|N u\|_{1}+\left(1+\left\|C_{0}\right\|_{*}\right) \sigma_{1}\right]+\|c\|_{1} .
\end{aligned}
$$

Therefore, it can be obtained that

$$
\|N u\|_{1} \leqslant \frac{\left.\left(\left\|a_{1}\right\|_{1}+\left\|b_{1}\right\|_{1}\right)\left(1+\left\|C_{0}\right\|_{*}\right) \sigma_{1}\right]+\|c\|_{1}}{1-\left(1+\left\|C_{0}\right\|_{*}+D\right)\left(\left\|a_{1}\right\|_{1}+\left\|b_{1}\right\|_{1}\right)} .
$$

From (29) and (28), one has

$$
\sup _{u \in \Omega_{1}}\|u\|=\sup _{u \in \Omega_{1}} \max \left\{\|u\|_{\infty},\left\|{ }^{c} D_{0+}^{\alpha-1} u\right\|_{\infty}\right\}<+\infty .
$$

Hence $\Omega_{1}$ is bounded in $X$. 
Set $\Omega_{2}=\{u \in \operatorname{Ker} L \mid N u \in \operatorname{Im} L\}$. Assuming $u \in \Omega_{2}$, one has $u=c_{2} t+C_{0} c_{2}$, $c_{2} \in \mathbb{R}^{n}$. Thus

$$
\begin{array}{rl}
C \int_{0}^{\eta}(\eta-s)^{\alpha-1} & f\left(s, c_{2} s+C_{0} c_{2}, c_{2}\right) d s-I \int_{0}^{1}(1-s)^{\alpha-1} f\left(s, c_{2} s+C_{0} c_{2}, c_{2}\right) d s \\
& =(I-C)(I-B)^{-1} B \int_{0}^{\tau}(\xi-s)^{\alpha-1} f\left(s, c_{2} s+C_{0} c_{2}, c_{2}\right) d s \in \operatorname{Im}(I-C) .
\end{array}
$$

Then, from assumption (M2), one has

$$
\begin{aligned}
\|u\| & =\max \left\{\|u\|_{\infty},\left\|{ }^{c} D_{0+}^{\alpha-1} u\right\|_{\infty}\right\} \\
& =\max \left\{\left\|c_{2} t+C_{0} c_{2}\right\|_{\infty},\left\|c_{2}\right\|_{\infty}\right\} \\
& \leqslant \max \left\{\left(1+\left\|C_{0}\right\|_{*}\right) \sigma_{1}, \sigma_{1}\right\} \\
& \leqslant\left(1+\left\|C_{0}\right\|_{*}\right) \sigma_{1}<+\infty
\end{aligned}
$$

Therefore, $\Omega_{2}$ is a bounded subset.

Set $\Omega_{3}^{ \pm}=\left\{u \in \operatorname{Ker} L: \pm \lambda_{1} u+\left(1-\lambda_{1}\right) Q N u=\theta, 0 \leqslant \lambda_{1} \leqslant 1\right\}$. We divide the proof into the following two steps:

Step 1 : For $u=c_{2} t+C_{0} c_{2} \in \Omega_{3}^{+}$, one has

$$
\lambda_{1}\left(c_{2} t+C_{0} c_{2}\right)+\left(1-\lambda_{1}\right) Q N\left(c_{2} t+C_{0} c_{2}\right)=\theta .
$$

Case 1 : If $\lambda_{1}=0$, then $Q N\left(c_{2} t+C_{0} c_{2}\right)=\theta$, such that $N\left(c_{2} t+C_{0} c_{2}\right) \in \operatorname{Ker} Q=\operatorname{Im} L$. Thus we have $N\left(c_{2} t+C_{0} c_{2}\right) \in \Omega_{2}$, so $\|u\| \leqslant\left(1+\left\|C_{0}\right\|_{*}\right) \sigma_{1}$.

Case 2 : If $\lambda_{1} \in(0,1]$, suppose $\|u\|>n \sigma_{2}$. Then, from (M3) obtain that

$$
0>-\lambda_{1}|u|^{2}=\left(1-\lambda_{1}\right)\langle u, Q N u\rangle \geqslant 0 .
$$

So, we have a contradiction. Thus $\|u\| \leqslant \sigma_{2}$.

Step 2 : For $u \in \Omega_{3}^{-}$, using same arguments as in Step 1 above, we can deduce that $\|u\| \leqslant \sigma_{2}$. Thus we can show that $\Omega_{3}^{-}, \Omega_{3}^{+} \subset X$ are two bounded subsets.

Now, let $\Omega \subset Y$ and $\bigcup_{i=1}^{3} \overline{\Omega_{i}} \subset \Omega$. According to the above arguments, we know that both conditions (i) and (ii) of Theorem 1 are satisfied. In order to prove (iii), we use isomorphic mapping $\mathcal{J}$ to construct the homotopy operator by

$$
H(x(t), \lambda)= \pm \lambda x(t)+(1-\lambda) \mathcal{J} Q N x(t) .
$$

Hence

$$
\begin{aligned}
\operatorname{deg}\left(\left.\mathcal{J} Q N\right|_{\text {Ker } L}, \Omega \cap \operatorname{Ker} L, \theta\right) & =\operatorname{deg}(H(\cdot, 0), \Omega \cap \operatorname{Ker} L, \theta) \\
& =\operatorname{deg}( \pm I d, \Omega \cap \operatorname{Ker} L, \theta) \neq 0 .
\end{aligned}
$$

Therefore, (iii) of Theorem 1 is satisfied. Theorem 2 is proved.

\section{Existence Results for Case (2)}

Now, we show the solvability of BVP (1), (2) when $B=I,|I-C|=0$. In this case, the boundary value condition degenerates to

$$
x(0)=x(\xi), \quad x(1)=C x(\eta) .
$$

Unlike Section 3, this section removes the restriction on matrix $C$ and uses the generalized inverse to conduct research under the most basic resonance conditions, inspired by [14]. 
Now we study the BVP (1) and (30) using Theorem 1. We use the same notations as in Section 3. $L, N, \mathcal{J}$. In this case,

$$
\operatorname{dom} L=\left\{x \in X_{1}: x \text { satisfies }(30)\right\} \text {. }
$$

Let $\mathcal{T}=I-C$ and $\mathcal{T}^{+}$be the Moore-Penrose pseudoinverse matrix of $\mathcal{T}$. From [24] we can get the following conclusions, which are necessary for our subsequent research:

(I $\left.I_{1}\right) \mathcal{T}^{+} \mathcal{T} \mathcal{T}^{+}=\mathcal{T}^{+}$

(I, $\mathcal{T} \mathcal{T}^{+} \mathcal{T}=\mathcal{T}$;

(I3) $\operatorname{Im} \mathcal{T}^{+} \mathcal{T}=\operatorname{Im} \mathcal{T}$

$\left(I_{4}\right) \operatorname{Im}\left(I-\mathcal{T}^{+} \mathcal{T}\right)=\operatorname{Ker} \mathcal{T}$.

From (12), we have

$$
\operatorname{Ker} L=\left\{c_{1}^{*} \in \mathbb{R}^{n}: \mathcal{T} c_{1}^{*}=\theta\right\} .
$$

Define a linear operator $H^{*}$ by

$$
H^{*} y(t)=\frac{\eta C-I}{\xi} I_{0+}^{\alpha} y(\xi)-C I_{0+}^{\alpha} y(\eta)+I_{0+}^{\alpha} y(1) .
$$

Then

$$
\operatorname{Im} L=\left\{y \in Y \mid H^{*} y(t) \in \operatorname{Im} \mathcal{T}\right\} .
$$

Define an operator $Q^{*}: Y \rightarrow Y$ as

$$
Q^{*} y=\gamma^{*} H^{*} y(t)
$$

where

$$
\gamma^{*}=\frac{\xi \alpha \Gamma(\alpha)}{\eta \xi^{\alpha}-\xi^{\alpha}+\xi-\xi \eta^{\alpha}}\left(I-\mathcal{T} \mathcal{T}^{+}\right)
$$

Then for $y \in Y$, we can get

$$
\begin{aligned}
Q^{* 2} y & =\gamma^{*} H^{*} Q^{*} y \\
& =\frac{\xi \alpha \Gamma(\alpha)}{\eta \xi^{\alpha}-\xi^{\alpha}+\xi-\xi \eta^{\alpha}}\left(I-\mathcal{T} \mathcal{T}^{+}\right) \frac{(\eta C-I) \xi^{\alpha}+\xi I-\xi \eta^{\alpha} C}{\alpha \xi \Gamma(\alpha)} Q^{*} y \\
& =Q^{*} y .
\end{aligned}
$$

In fact

$$
\begin{aligned}
\left(I-\mathcal{T} \mathcal{T}^{+}\right)(\eta C-I) \xi^{\alpha}+\xi I-\xi \eta^{\alpha} C \\
\left.\quad=\left(I-\mathcal{T} \mathcal{T}^{+}\right)\left\{\eta(C-I) \xi^{\alpha}+(\eta-1) \xi^{\alpha} I+\eta^{\alpha}(I-C)+\left(1-\eta^{\alpha}\right) I\right)\right\} \\
\quad=(\eta-1) \xi^{\alpha} I+\xi\left(1-\eta^{\alpha}\right)\left(I-\mathcal{T} \mathcal{T}^{+}\right) .
\end{aligned}
$$

By similar arguments to Lemma 2.5 in [14], we have that the index of the Fredholm operator $L$ is zero.

Define an operator $P^{*}: X \rightarrow X$ as

$$
P^{*} x(t)=\left(I-\mathcal{T}^{+} \mathcal{T}\right) x(0)
$$

If $v \in \operatorname{Ker} L$, one has $v=c_{1}^{*}, c_{1}^{*} \in \mathbb{R}^{n} \cap \operatorname{Ker}(\mathcal{T})=\operatorname{Im}\left(I-\mathcal{T}^{+} \mathcal{T}\right)$, thus there exists $d_{1}^{*} \in \mathbb{R}^{n}$ suct that

$$
c_{1}^{*}=\left(I-\mathcal{T}^{+} \mathcal{T}\right) d_{1}^{*} .
$$

So, $v \in \operatorname{Im} P^{*}$. Conversely, if $v \in \operatorname{Im} P^{*}$, from $\left(I_{2}\right)$ we can know that $v \in \operatorname{Ker} \mathcal{T}$. Again, since Ker $P^{*} \cap \operatorname{Ker} L=\{\theta\}$, then $X=\operatorname{Ker} P^{*} \oplus \operatorname{Ker} L$.

Define a mapping $K_{P}^{*}: \operatorname{Im} L \rightarrow \operatorname{Ker} P^{*} \cap \operatorname{dom} L$ as

$$
K_{P}^{*} y(s)=\mathcal{T}^{+} H^{*} y+I_{0+}^{\alpha} y(s)-\frac{s}{\xi} I_{0+}^{\alpha} y(\xi)
$$


Through checking calculation, we can get $K_{P}^{*} y \in \operatorname{dom} L$ and $K_{P}^{*} y \in \operatorname{Ker} P^{*}$. Thus the definition of $K_{P}^{*}$ is reasonable.

Letting $u \in \operatorname{Ker} P^{*} \cap \operatorname{dom} L$, one has

$$
\begin{aligned}
K_{P}^{*} L u(t) & =\mathcal{T}^{+} H^{* c} D_{0+}^{\alpha} u+I_{0+}^{\alpha}{ }^{c} D_{0+}^{\alpha} u(t)-\frac{t}{\xi} I_{0+}^{\alpha}{ }^{c} D_{0+}^{\alpha} u(\xi) \\
& =-\mathcal{T}^{+}(\eta C-I) u^{\prime}(0) \xi-\mathcal{T}^{+} C\left(u(0)+u^{\prime}(0) \eta\right)+\mathcal{T}^{+}\left(u(0)+u^{\prime}(0)\right)+u(t)-u(0) \\
& =-\mathcal{T}^{+} C u^{\prime}(0) \eta+\mathcal{T}^{+} u^{\prime}(0)+\mathcal{T}^{+}((\eta C-I)) u^{\prime}(0)-\left(I-\mathcal{T}^{+} \mathcal{T}\right) u(0)+u(t) \\
& =u(t) .
\end{aligned}
$$

Similarly, for $y \in \operatorname{Im} L$, we have $L K_{P}^{*} y=y$. Then we can deduce that $K_{P}^{*}=\left(\left.L\right|_{\operatorname{dom} L \cap \operatorname{Ker} P}\right)^{-1}$. Denote

$$
D^{*}=2+\left\|\mathcal{T}^{+}\right\|_{*}\left((\eta+1)\|C\|_{*}+2\right) .
$$

By the similar proof process as in Lemma 4 and Lemma 5, we know that $\left\|K_{P}^{*} y\right\| \leqslant D^{*}\|y\|_{1}$, and $K_{P}^{*}(I-Q) N$ is completely continuous.

Now we give the following assumptions:

$\left(\mathrm{M}^{*}\right)$ For all $s \in[0,1], u, v \in \mathbb{R}^{n}$, we have

$$
|f(s, u, v)| \leqslant a|u|+b|v|+c,
$$

where $a, b, c \in Y$ are three positive functions satisfying $\left(\left\|I-\mathcal{T} \mathcal{T}^{+}\right\|_{*}+D^{*}\right)\left(\|a\|_{1}+\right.$ $\left.\|b\|_{1}\right)<1$, and $D^{*}$ is the constant given in (34).

(M2*) For all $u \in \operatorname{dom} L$, if

$$
H^{*} f\left(s, u(t),{ }^{c} D_{0+}^{\alpha-1} u(t)\right) \in \operatorname{Im}(\mathcal{T}) .
$$

Then, there exist $\sigma_{1}^{*} \in R_{+}$and $s_{0} \in[0,1]$, s.t. $\left|u\left(s_{0}\right)\right| \leqslant \sigma_{1}^{*}$.

(M3*) There exist $\sigma_{2}^{*} \in R_{+}$, s.t. for every $v \in \mathbb{R}^{n}$ with $v=C v$ and $|v|>\sigma_{2}^{*}$, either

$$
\left\langle v, Q^{*} N(v)\right\rangle \leqslant 0 \text { or }\left\langle v, Q^{*} N(v)\right\rangle \geqslant 0,
$$

where $\langle\cdot, \cdot\rangle$ stands for scalar product in $\mathbb{R}^{n}$.

Theorem 3. If assumptions $\left(M 1^{*}\right)-\left(M 3^{*}\right)$ are satisfied, BVP (1) and (30) has at least one solution in $X$.

Proof. We use the same definitions of $\Omega_{1}, \Omega_{2}$, and $\Omega_{3}$ as in Theorem 2 .

For $x \in \Omega_{1}$, we have that $N x \in \operatorname{Im} L=\operatorname{Ker} Q^{*}$. Similarly, we can show

$$
H^{*} f\left(s, u(t),{ }^{c} D_{0+}^{\alpha-1} u(t)\right) \in \operatorname{Im}(\mathcal{T}) .
$$

In fact,

$$
\begin{aligned}
H^{*} f\left(s, u,{ }^{c} D_{0+}^{\alpha-1} u\right) & =H^{* c} D_{0+}^{\alpha} u \\
& =(\eta C-I) u^{\prime}(0)+C u(\eta)+u(0)-C u(0)+(I-C \eta) u^{\prime}(0)-u(1) \\
& =\mathcal{T} u(0) \in \operatorname{Im}(\mathcal{T}) .
\end{aligned}
$$

Using assumption $\left(\mathrm{M} 2^{*}\right)$, we can deduce that

$$
|u(0)|=\left|u\left(t_{0}\right)-\int_{0}^{t_{0}}{ }^{c} D_{0+}^{\alpha-1} u(s) d s\right| \leqslant \sigma_{1}^{*}+\left\|{ }^{c} D_{0+}^{\alpha-1} u\right\|_{\infty},
$$

and

$$
\left|{ }^{c} D_{0+}^{\alpha-1} u(t)\right| \leqslant \int_{0}^{t}\left|{ }^{c} D_{0+}^{\alpha} u(s)\right| d s \leqslant\|L u\|_{1} .
$$


Then with the similar proof process in Theorem 2 we can know that

$$
\|u(t)\| \leqslant\left(\left\|I-\mathcal{T}^{+} \mathcal{T}\right\|_{*}+D^{*}\right)\|N u\|_{1}+\sigma_{1}^{*}\left\|I-\mathcal{T}^{+} \mathcal{T}\right\|_{*},
$$

and

$$
\|N u\|_{1} \leqslant \frac{\sigma_{1}^{*}\left(\|a\|_{1}+\|b\|_{1}\right)\left\|I-\mathcal{T}^{+} \mathcal{T}\right\|_{*}+\|c\|_{1}}{-\left(\left\|I-\mathcal{T}^{+} \mathcal{T}\right\|_{*}+D^{*}\right)\left(\|a\|_{1}+\|b\|_{1}\right)+1} .
$$

Combining (38) and (39) we can deduce that

$$
\sup _{x \in \Omega_{1}}\|u\|=\sup _{u \in \Omega_{1}} \max \left\{\|u\|_{\infty},\left\|{ }^{c} D_{0+}^{\alpha-1} u\right\|_{\infty}\right\}<+\infty .
$$

Hence $\Omega_{1}$ is a bounded subset of $X$.

For $u \in \Omega_{2}$, one has $u=c_{1}^{*}, c_{1}^{*} \in \mathbb{R}^{n}$. Combining with $N u \in \operatorname{Im} L$, we can get

$$
H^{*} N u \in \operatorname{Im}(\mathcal{T})
$$

From assumptions $(\mathrm{M} 2 *)$, we get

$$
\|u\|=\max \left\{\|u\|_{\infty},\left\|{ }^{c} D_{0+}^{\alpha-1} u\right\|_{\infty}\right\}=\|c\|_{\infty}=\left|u\left(t_{0}\right)\right| \leqslant \sigma_{1}^{*}<+\infty .
$$

Such that $\Omega_{2}$ is bounded in $X$.

In order to prove both $\Omega_{3}^{-}$and $\Omega_{3}^{+}$are bounded, we also divide the proof process into two steps:

Step 1 : Assuming $u \in \Omega_{3}^{-}$, one has $u=c_{1}^{*}$, where $c_{1}^{*} \in \mathbb{R}^{n} \cap \operatorname{Ker}(\mathcal{T})$. Thus we have

$$
-\lambda c_{1}^{*}+(1-\lambda) Q N\left(c_{1}^{*}\right)=\theta .
$$

Case 1 : If $\lambda=0$, then $Q N\left(c_{1}^{*}\right)=\theta$, such that $N\left(c_{1}^{*}\right) \in \operatorname{Ker} Q=\operatorname{Im} L$. Thus we have $N x \in \Omega_{2}$, so $\|x\| \leqslant \sigma_{1}^{*}$.

Case 2 : If $\lambda \in(0,1]$, suppose $\|u\|>\sigma_{2}$. From (B3) we get

$$
0<\lambda\left|c_{1}^{*}\right|^{2}=(1-\lambda)\left\langle c_{1}^{*}, Q N c_{1}^{*}\right\rangle \leqslant 0 .
$$

Therefore, we have $\|u\| \leqslant \sigma_{2}^{*}$.

Step 2 : For $u \in \Omega_{3}^{+}$, through a similar proof process as in Step 1, we can deduce that $\|u\| \leqslant \sigma_{2}^{*}$.

Thus, $\Omega_{3}^{-}$and $\Omega_{3}^{+}$are two bounded subsets in $X$.

Let the definitions of bounded open subset $\Omega$ and homotopy $H(u, \lambda)$ be the same as in Theorem 2. Then we can deduce that (iii) of Theorem 1 is also satisfied. By Theorem 1, Equations (1) and (30) must have a solution in $\operatorname{dom} L \cap \Omega$.

\section{Examples}

In this section, we present two examples to illustrate our main results in Sections 3 and 4.

Example 1. Consider the following boundary value problem:

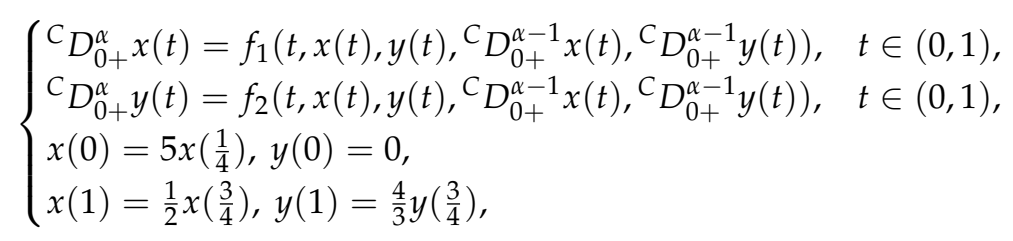

where $\alpha=\frac{3}{2}, f_{i}:[0,1] \times \mathbb{R}^{4} \rightarrow \mathbb{R}, i=1,2$, are defined as

$$
f_{1}\left(t, x_{1}, x_{2}, y_{1}, y_{2}\right)=-\frac{x_{1}+y_{1}}{40}
$$




$$
f_{2}\left(t, x_{1}, x_{2}, y_{1}, y_{2}\right)=\frac{\left|x_{2}\right|+\left|y_{2}\right|+1}{60}
$$

for all $t \in[0,1]$.

Clearly, $\xi=\frac{1}{4}, \eta=\frac{3}{4}$

$$
B=\left[\begin{array}{ll}
5 & 0 \\
0 & 0
\end{array}\right], C=\left[\begin{array}{cc}
\frac{1}{2} & 0 \\
0 & \frac{4}{3}
\end{array}\right],(I-C)(I-B)^{-1} B=\left[\begin{array}{cc}
-\frac{5}{8} & 0 \\
0 & 0
\end{array}\right], C_{0}=\left[\begin{array}{cc}
-\frac{5}{16} & 0 \\
0 & 0
\end{array}\right],
$$

and $I-\eta C+\xi(I-C)(I-B)^{-1} B=\Theta$. Denote $u_{1}=\left(x_{1}, x_{2}\right), u_{2}=\left(y_{1}, y_{2}\right) \in \mathbb{R}^{2}$, define function $f:[0,1] \times \mathbb{R}^{2} \times \mathbb{R}^{2} \rightarrow \mathbb{R}^{2}$

$$
f\left(t, u_{1}, u_{2}\right)=\left(f_{1}\left(t, u_{1}, u_{2}\right), f_{2}\left(t, u_{1}, u_{2}\right)\right)^{\top}, \forall t \in[0,1] .
$$

By (41), (42), and (43), $f$ satisfies Carathéodory conditions.

Now we show that the other conditions of Theorem 3 hold. Choose positive integrable functions

$$
a(t)=b(t)=c(t)=\frac{1}{40} .
$$

Then we have

$$
|f(t, u, v)| \leqslant a(t)|u|+b(t)|v|+c(t),
$$

By some simple computation, we get

$$
\left(1+\left\|C_{0}\right\|_{*}+D\right)\left(\|a\|_{1}+\|b\|_{1}\right)=\frac{25}{384}<1 .
$$

Hence, (M1) is satisfied.

In order to check (M2), one has

$$
f_{2}\left(t, u(t),{ }^{C} D_{0+}^{\alpha-1} u(t)\right)>\frac{1}{60},
$$

for all $u \in C^{1}\left([0,1] ; \mathbb{R}^{2}\right)$ and all $t \in[0,1]$. Letting $f_{2}\left(t, u(t),{ }^{C} D_{0+}^{\alpha-1} u(t)\right)=f_{2}$ be a positive constant, we have

$$
\begin{aligned}
C \int_{0}^{\eta}(\eta-s)^{\alpha-1} f\left(t, u(t),{ }^{C} D_{0+}^{\alpha-1} u(t)\right) d t \\
\quad-I \int_{0}^{1}(1-s)^{\alpha-1} f\left(t, u(t),{ }^{C} D_{0+}^{\alpha-1} u(t)\right) d t=\left[\begin{array}{c}
\frac{1}{2} f_{1}^{*}+f_{2}^{*} \\
\frac{260}{2911} f_{2}
\end{array}\right],
\end{aligned}
$$

where $f_{1}^{*}=I_{0+}^{\alpha} f_{1}(\eta), f_{2}^{*}=I_{0+}^{\alpha} f_{1}(1)$. If $f_{2}=\frac{1}{60}$, there is

$$
\begin{aligned}
C \int_{0}^{\eta}(\eta-s)^{\alpha-1} f\left(t, u(t),{ }^{C} D_{0+}^{\alpha-1} u(t)\right) d t \\
\quad-I \int_{0}^{1}(1-s)^{\alpha-1} f\left(t, u(t),{ }^{C} D_{0+}^{\alpha-1} u(t)\right) d t=\left[\begin{array}{c}
\frac{1}{2} f_{1}^{*}+f_{2}^{*} \\
\frac{13}{8733}
\end{array}\right] .
\end{aligned}
$$

This shows that when $f_{2}\left(t, u(t),{ }^{C} D_{0+}^{\alpha-1} u(t)\right)>f_{2}=\frac{1}{60}$, one has

$$
\begin{aligned}
C \int_{0}^{\eta}(\eta-s)^{\alpha-1} f\left(t, u(t),{ }^{C} D_{0+}^{\alpha-1} u(t)\right) d t \\
\quad-I \int_{0}^{1}(1-s)^{\alpha-1} f\left(t, u(t),{ }^{C} D_{0+}^{\alpha-1} u(t)\right) d t \notin \operatorname{Im}\left((I-C)(I-B)^{-1} B\right),
\end{aligned}
$$

because $\operatorname{Im}\left((I-C)(I-B)^{-1} B\right)=\left\{(p, 0)^{\top}: p \in \mathbb{R}\right\}$. 
Finally, we check (M3). Let $q(t)=\left(t I+C_{0}\right)$. Denote $\tau=\left(\tau_{1}, \tau_{2}\right)^{\top}$. So

$$
\begin{gathered}
q(t) \tau=\left(\left(t+\frac{5}{16}\right) \tau_{1}, t \tau_{2}\right)^{\top} \\
{ }^{C} D_{0+}^{\alpha-1} q(t)=\left(\frac{2 \tau_{1} \sqrt{t}}{\sqrt{\pi}}, \frac{2 \tau_{2} \sqrt{t}}{\sqrt{\pi}}\right)^{\top} .
\end{gathered}
$$

Then there is

$$
N q(t) \tau=\left(-\frac{\left(t+\frac{5}{16}\right) \tau_{1}}{40}-\frac{2 \tau_{1} \sqrt{t}}{40 \sqrt{\pi}}, \frac{t\left|\tau_{2}\right|+\frac{2 \sqrt{t}}{\sqrt{\pi}}\left|\tau_{2}\right|+1}{60}\right)^{\top}
$$

So

$$
Q N(q(t) \tau)=\alpha\left[\begin{array}{c}
-\frac{31}{5234} \tau_{1} \\
\frac{14}{34946}\left|\tau_{2}\right|+\frac{25}{181937}
\end{array}\right]
$$

and

$$
\langle q(t) \tau, Q N(q(t) \tau)\rangle=\alpha\left(-\frac{31}{5234} \tau_{1}^{2}+\frac{15}{34946}\left|\tau_{2}\right| \tau_{2}+\frac{25}{181937} \tau_{2}\right) \leqslant 0 .
$$

In fact, if $\tau_{2} \leqslant 0$, this is obviously true. If $\tau_{2}>0$, letting $\left|\tau_{2}\right| \geq 1$, one has $\tau_{2}^{2}>\tau_{2}$. Again, since

$$
\frac{15}{34946}>\frac{25}{181937} \text {. }
$$

So, the formula above has no real root, which means $-\frac{31}{5234} \tau_{1}^{2}+\frac{15}{34946}\left|\tau_{2}\right| \tau_{2}+\frac{25}{181937} \tau_{2}<0$. Thus, by Theorem 2, BVP (40) has at least one solution.

Example 2. Consider the following boundary value problem:

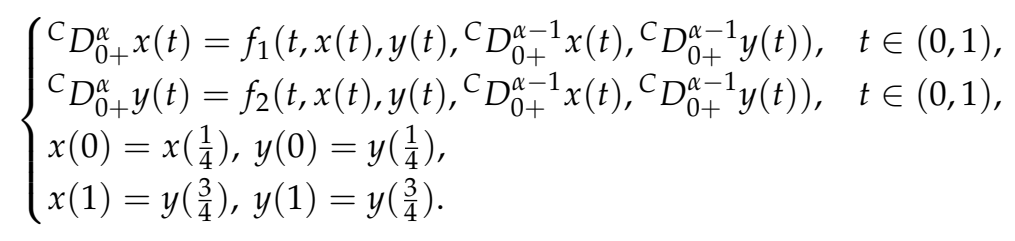

We use the same $\alpha, f, \xi, \eta, a(t), b(t)$, and $c(t)$ as in Example 1 and $f_{i}:[0,1] \times \mathbb{R}^{4} \rightarrow$ $\mathbb{R}, i=1,2$ are defined as

$$
\begin{gathered}
f_{1}\left(t, x_{1}, x_{2}, y_{1}, y_{2}\right)=\frac{x_{2}+y_{2}}{40} . \\
f_{2}\left(t, x_{1}, x_{2}, y_{1}, y_{2}\right)= \begin{cases}\frac{\sqrt{y_{1}^{2}+y_{2}^{2}}}{40}, & \text { if }\left|u_{2}\right|>1 ; \\
f_{1}\left(t, x_{1}, x_{2}, y_{1}, y_{2}\right), & \text { otherwise. }\end{cases} \\
C=\left[\begin{array}{ll}
0 & 1 \\
0 & 1
\end{array}\right], \mathcal{T}=\left[\begin{array}{cc}
1 & -1 \\
0 & 0
\end{array}\right], \mathcal{T}^{+}=\left[\begin{array}{cc}
\frac{1}{2} & 0 \\
-\frac{1}{2} & 0
\end{array}\right] .
\end{gathered}
$$

We can easily check that assumption (M1*) is satisfied. When $\left|u_{2}\right|>1$, from the definition of $f$, one has $y_{1}^{2}+y_{2}^{2}>1$ and $H^{*} f_{2}>H^{*} \frac{1}{40}=\frac{51}{13571}>0$. According to a similar proof process as Example 1, one has

$$
H^{*} f\left(t, u(t),{ }^{C} D_{0+}^{\alpha-1} u(t)\right)=\left[\begin{array}{l}
H^{*} f_{1} \\
H^{*} f_{2}
\end{array}\right] \notin \operatorname{Im}(\mathcal{T}),
$$


because $\operatorname{Im}(\mathcal{T})=\left\{(p, 0)^{\top}: p \in \mathbb{R}\right\}$. Finally, we check (M3*). Letting $\tau=\left(\tau_{0}, \tau_{0}\right)^{\top} \in$ $\operatorname{Ker}(\mathcal{T})$, one has

$$
N \tau=\left(f_{1}(t, \tau, \theta), f_{2}(t, \tau, \theta)\right)^{\top}= \begin{cases}\left(\frac{\tau_{0}}{44}, 0\right)^{\top}, & \text { if }\left|u_{2}\right|>1 \\ \left(\frac{\tau_{0}}{40}, \frac{\tau_{0}}{40}\right)^{\top}, & \text { otherwise. }\end{cases}
$$

So

$$
\begin{aligned}
& Q N \tau \\
& =\gamma^{*} \begin{cases}{\left[\begin{array}{c}
\tau_{0} \\
120 \\
0
\end{array}\right],} & \text { if }\left|u_{2}\right|>1 ; \\
{\left[\begin{array}{l}
\left(7 \tau_{0}\right) / 480-\left(3^{1 / 2} \tau_{0}\right) / 160 \\
\left(7 \tau_{0}\right) / 480-\left(3^{1 / 2} \tau_{0}\right) / 160
\end{array}\right],} & \text { otherwise, }\end{cases}
\end{aligned}
$$

and

$$
\langle\tau, Q N \tau\rangle= \begin{cases}\frac{1}{120} \tau_{0}^{2} c, & \text { if }\left|u_{2}\right|>1 \\ \frac{7-3 * 3^{1 / 2}}{480} \tau_{0}^{2} c, & \text { otherwise }\end{cases}
$$

where $c=\frac{\xi \alpha \Gamma(\alpha)}{\eta \xi^{\alpha}-\tilde{\zeta}^{\alpha}+\xi^{\xi}-\xi^{\alpha}}=\frac{5302 \pi^{1 / 2}}{1594}>0$. Thus, $\langle\tau, Q N \tau\rangle>0$, by Theorem 3, (43) has at least one solution.

\section{Conclusions}

This paper mainly studied a class of second-order nonlocal boundary value problem systems at resonance which state variable $x \in \mathbb{R}^{n}$, and gave two new theorems on the existence of solutions in different kernel spaces by using the Mawhin coincidence degree theorem.

In the future, we could consider studying resonance boundary value problems under less-restricted conditions or under more complicated boundary value conditions.

Author Contributions: Conceptualization, Y.F. and Z.B. All authors have read and agreed to the published version of the manuscript.

Funding: This research was funded by NSFC grant number 11571207 and Shandong Provincial Natural Science Foundation number ZR2021MA064 and the Taishan Scholar project.

Institutional Review Board Statement: Not applicable.

Informed Consent Statement: Not applicable.

Data Availability Statement: Not applicable.

Conflicts of Interest: The authors declare no conflict of interest.

\section{References}

1. Il'in, V.A.; Moiseev, E.I. Nonlocal boundary value problem of the first kind for a Sturm-Liuville operator in its differential and finite difference aspects. Differ. Uravn. 1987, 23, 803-810.

2. Il'in, V.A.; Moiseev, E.I. Nonlocal boundary value problem of the second kind for a Sturm-Liuville operator. Differ. Uravn. 1987, 23, 979-987.

3. Bitsadze, A.V.; Samarskogo, A.A. On some simplest generalizations of linear elliptic boundary value problems. Rep. Acad. Sci. $1969,185,739-740$.

4. Gupta, C.P. A sharper condition for the solvability of a three-point second order boundary value problem. J. Math. Anal. Appl. 1997, 205, 579-586. [CrossRef]

5. Cui, Y.J. Solvability of second-order boundary-value problems at resonance involving integral conditions. Electron. J. Differ. Equ. 2012, 45, 1-9.

6. Bai, Z.B.; Li, W.G.; Ge, W.G. Existence and multiplicity of solutions for four-point boundary value problems at resonance. Nonlinear Anal. 2005, 60, 1151-1162. [CrossRef]

7. Kosmatov, N. A singular non-local problem at resonance. J. Math. Anal. Appl. 2005, 394, 425-431. [CrossRef] 
8. Feng, W.; Webb, J.R.L. A singular non-local problem at resonance. Nonlinear Anal. 1997, 30, 3227-3238. [CrossRef]

9. Ma, R.Y. Multiplicity results for a three-point boundary value problem at resonance. Nonlinear Anal. 1997, 53, 777-789. [CrossRef]

10. Gupta, C.P. Existence theorems for a second order m-point boundary value problem at resonance. Internat. J. Math. Math. Sci. 1997, 53, 777-789.

11. Liu, B. Solvability of multi-point boundary value problem at resonance-Part IV. Appl. Math. Comput. 2003, 143, 275-299. [CrossRef]

12. Zhang, Y.H.; Bai, Z.B.; Feng, T.T. Existence results for a coupled system of nonlinear fractional three-point boundary value problems at resonance. Comput. Math. Appl. 2011, 61, 1032-1047. [CrossRef]

13. Phung, P.D.; Truong, L.X. On the existence of a three point boundary value problem at resonance in $\mathbb{R}^{n}$. J. Math. Anal. Appl. 2014, 416, 522-533. [CrossRef]

14. Phung, P.D.; Truong, L.X. Existence of solutions to three-point boundary-value problems at resonance. Electron. J. Diff. Eqns. 2016, $115,1-13$

15. Phung, P.D.; Minh, H.B. Existence of solutions to fractional boundary value problems at resonance in Hilbert spaces. Bound. Value Probl. 2017, 2017, 105. [CrossRef]

16. Phung, P.D. Solvability of multipoint BVPs at resonance for various kernels. J. Sci. Technol. Food. 2020, 20, 3-16.

17. Jiang, W.H.; Yang, C.X. Existence of positive solutions for multi-point resonance systems of differential equations with boundary value conditions. J. Hebei Univ. Sci. Tech. 2016, 37, 340-348.

18. Mawhin, J.; Szymańska-Debowska, K. Convexity, topology and nonlinear differential systems with nonlocal boundary conditions: A survey. Rend. Istit. Mat. Univ. 2019, 51, 125-166.

19. Mawhin, J.; Szymańska-Debowska, K. Existence of solutions for fractional differential equations with three-point boundary conditions at resonance in $\mathbb{R}^{n}$. Electron. J. Qual. Theory Differ. 2014, 68, 1-18.

20. He, B.B. Existence of solutions to fractional differential equations with three-point boundary conditions at resonance with general conditions. Fract. Calc. Appl. 2018, 9, 120-136.

21. Zhou, H.C.; Ge, F.D.; Kou, C.H. Existence of solutions to fractional differential equations with multi-point boundary conditions at resonance in Hilbert spaces. Electron. J. Differ. Equ. 2016, 61, 1-16.

22. Kilbas, A.A.; Srivastava, H.M.; Trujillo, J.J. Theory and Applications of Fractional Differential Equations; North-Holland Mathematics Studies; Elsevier: Amsterdam, The Netherlands, 2006; Volume 204.

23. Mawhin, J. Topological Degree Methods in Nonlinear Boundary Value Problems, 1st ed.; American Mathematical Society: Providence, RI, USA, 1979.

24. Ben-Israel, A.; Greville, T.N.E. Generalized Inverses: Theory and Applications, 1st ed.; Springer: New York, NY, USA, 2003. 